\title{
OPEN Validation and application of a novel in vivo cervical spine kinematics analysis technique
}

\author{
Zongmiao Wan ${ }^{1,6}$, Wenjin Wang ${ }^{2,3,6}$, Chao Li ${ }^{1}$, Junjie $\mathrm{Li}^{2}$, Jinpeng Lin ${ }^{2}$, Fei Tian ${ }^{2,4}$, Ting Zhu' ${ }^{2}$, \\ Danni Wu ${ }^{2}$, Luqi Guo ${ }^{2}$ \& Shaobai Wang ${ }^{2,5}$
}

To validate the accuracy of Cone beam computed tomography (CBCT) cervical spine modeling with three dimensional (3D)-3D registration for in vivo measurements of cervical spine kinematics. CBCT model accuracy was validated by superimposition with computed tomography (CT) models in 10 healthy young adults, and then cervical vertebrae were registered in six end positions of functional movements, versus a neutral position, in 5 healthy young adults. Registration errors and six degrees of freedom (6-DOF) kinematics were calculated and reported. Relative to CT models, mean deviations of the CBCT models were $<0.6 \mathrm{~mm}$. Mean registration errors between end positions and the reference neutral position were $<0.7 \mathrm{~mm}$. During flexion-extension (F-E), the translation in the three directions was small, mostly $<1 \mathrm{~mm}$, with coupled $L B$ and $A R$ both $<1^{\circ}$. During lateral bending (LB), the bending was distributed roughly evenly, with coupled axial rotation (AR) opposite to the LB at C1-C2, and minimal coupled F-E. During AR, most of the rotation occurred in the $C 1-C 2$ segment $\left(29.93 \pm 7.19^{\circ}\right.$ in left twist and $31.38 \pm 8.49^{\circ}$ in right twist) and coupled $L B$ was observed in the direction opposite to that of the AR. Model matching demonstrated submillimeter accuracy in cervical spine kinematics data. The presently evaluated low-radiation-dose $C B C T$ technique can be used to measure 3D spine kinematics in vivo across functional $F-E$, $A R$, and $L B$ positions, which has been especially challenging for the upper cervical spine.

The lifestyle changes that come with nation development have been associated with increased incidences and earlier onset of cervical spine pathology ${ }^{1-3}$. Accurate measurement of spine kinematics is helpful for characterizing intervertebral changes, investigating biomechanical mechanisms of cervical spine pathology, and evaluating surgical outcomes ${ }^{4-9}$, including assessing the effects of vertebral fusion on the mobility of adjacent vertebrae ${ }^{10}$.

Traditionally, kinematic analyses of the cervical spine have been based on cadaveric simulations and on twoand three-dimensional (2D and 3D) images. Cadaveric studies cannot reproduce joint motion accurately due to the lack of muscular activities and a physiological environment ${ }^{11}$. The main limitation of $2 \mathrm{D}$ images, such as radiographs ${ }^{10,11}$, is lack of information about $3 \mathrm{D}$ movement characteristics ${ }^{14,15}$. Although computed tomography (CT) and magnetic resonance imaging (MRI) techniques can be used to obtain $3 \mathrm{D}$ in vivo measurements ${ }^{16,17}$, they are performed in subjects that are lying down and thus without physiological loading. Additionally, MRI, $\mathrm{CT}$, and fluoroscopy have the respective drawbacks of a long scanning time, substantial radiation exposure, and motion artifacts. Cone beam computed tomography (CBCT) is a state-of-the-art imaging technique that provides diagnostic quality images with reasonably low radiation dose scanning protocols. The generation of $3 \mathrm{D}$ reconstructions of cervical spine structures were reported in several studies ${ }^{18-20}$.

Quantitative knowledge of in vivo cervical spine kinematics is important for understanding cervical spine pathology and for improving surgical treatment of cervical spine degenerative disease. The purpose of the present study was to validate the accuracy of CBCT modeling and a 3D-3D registration technique for the measurement of in vivo cervical spine kinematics. We hypothesize that the technique can be used for segmental kinematic analysis of the cervical spines, especially at the end positions of functional movements.

\footnotetext{
${ }^{1}$ Department of Orthopedics, The First Affiliated Hospital of Nanchang University, Nanchang 330000, Jiangxi, China. ${ }^{2}$ Key Laboratory of Exercise and Health Sciences of Ministry of Education, School of Kinesiology, Shanghai University of Sport, Shanghai 20043, China. ${ }^{3}$ Institute of Biomechanics and Orthopaedics, German Sport University Cologne, 50933 Cologne, Germany. ${ }^{4}$ Department of Rehabilitation Medicine, Heping Hospital Affiliated To Changzhi Medical College, Shanxi 046000, China. ${ }^{5}$ Present address: School of Kinesiology, Shanghai University of Sport, Research Building 412, 200 Hengren Road, Shanghai 200438, China. ${ }^{6}$ These authors contributed equally: Zongmiao Wan and Wenjin Wang. ${ }^{\square}$ email: wangs@innomotion.biz
} 

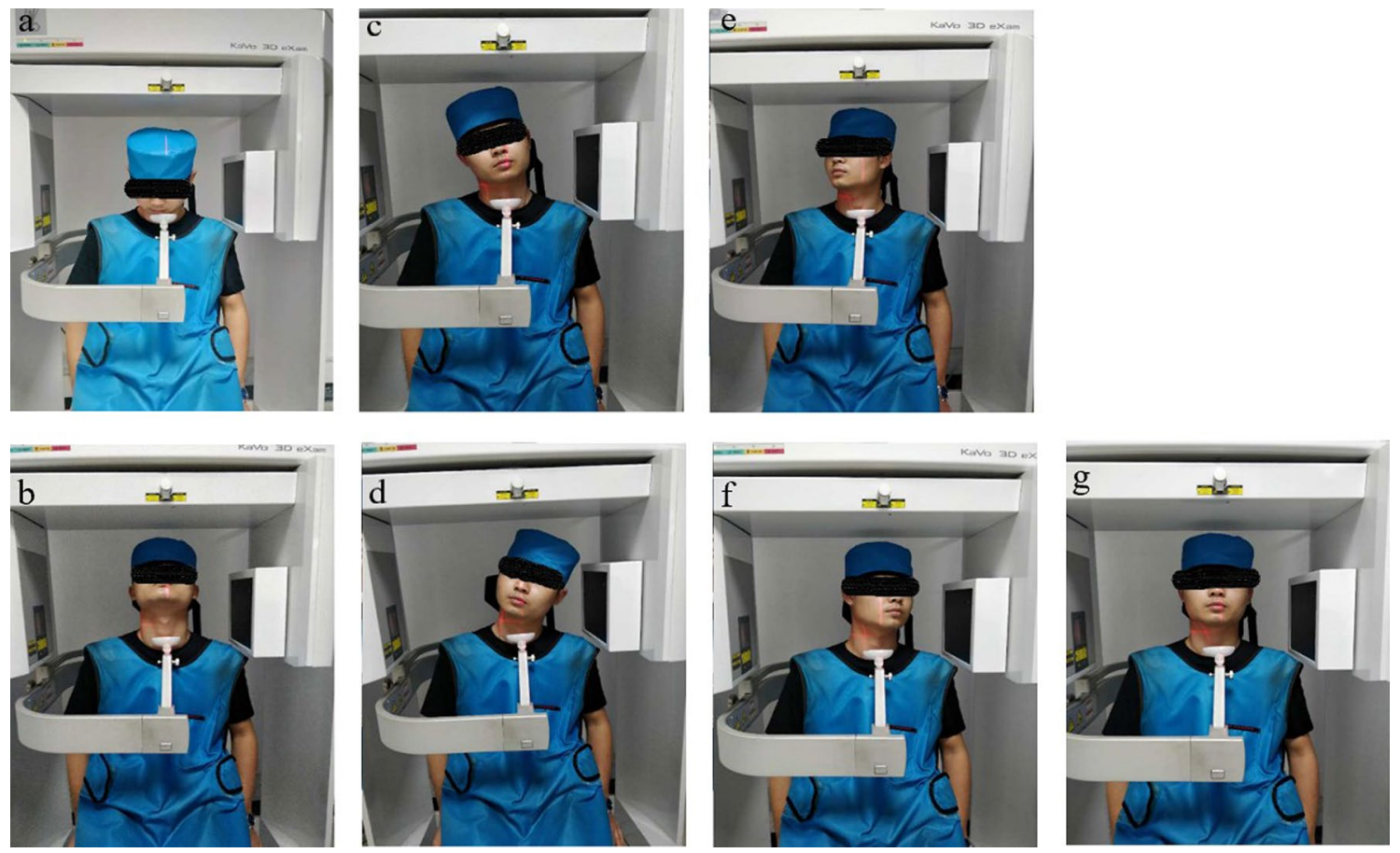

Figure 1. Overview of cone-beam computed tomography (CBCT) scanning showing a participant performing $(\mathbf{a}, \mathbf{b})$ flexion-extension (F-E), (c,d) lateral bending (LB), and (e,f) axial rotation (AR) movements, as well as ( $\mathbf{g})$ a neutral position.

\section{Methods}

The validation and application of 3D-3D registration technique for in vivo measurement of cervical spine kinematics was conducted in three phases. In the first phase, we validated our CBCT models' accuracy by superimposing them with corresponding CT models. In the second phase, cervical vertebrae were registered in six end positions of the movement relative to a corresponding neutral position. In the third phase, we applied this method to obtain measurements over six degrees of freedom (6-DOF) of both the upper and subaxial cervical spine vertebrae.

Participants. A group of 10 healthy young adults (5 women and 5 men) with a mean age ( \pm standard deviation) of $30.20 \pm 6.11$ years participated in the first phase of the study. Subsequently, 5 healthy young adults (3 women and 2 men) with a mean age of $27.8 \pm 6.7$ years, who did not participate in phase 1, participated in the second and third phases. All participants signed an informed consent form prior to participation, and identifying information/images have been obtained informed consent. The study protocol was approved by the institutional review board at the First Affiliated Hospital of Nanchang University (2020, no. 46), and all methods were performed in accordance with the relevant guidelines and regulations. Radiation safety approval was obtained.

Data acquisition. In the first phase, each participant received a CT (SOMATOM Definition AS+) and a CBCT (I-CAT, KaVo3DeXam, American) scan. The CT parameters were: slice thickness, $0.6 \mathrm{~mm}$; pixel size, $0.25 \mathrm{~mm}$; tube current, $190 \mathrm{~mA} ; 130 \mathrm{kV}$; and scan time, $8.6 \mathrm{~s}$. The CBCT parameters were: slice thickness, $0.2 \mathrm{~mm}$; pixel size, $0.25 \mathrm{~mm}$; tube current, $20.27 \mathrm{~mA}$, voltage, $120 \mathrm{kV}$; and rotation (scan) time $14.7 \mathrm{~s}$. The CT and CBCT datasets were used to construct surface models of each subject's vertebrae via segmentation and reconstruction processed using solid modeling software (Amira 6.7.0 Thermo Fisher Scientific, Rockford, IL).

In the second and third phases, each participant received only CBCT scans. The CBCT datasets were used to construct surface models of each subject's vertebrae via segmentation and reconstruction processed using solid modeling software (Amira). The participants performed active functional head flexion-extension (F-E), functional lateral bending (LB) in which the head was bent down leftward and then rightward, functional axial rotation (AR) in which the head was rotated to the left and the right, and neutral tasks while sitting on a chair with a stabilized trunk, wearing a lead apron to protect the lower body from radiation (Figs. 1 and 2). Data for all seven positions were collected in a single trial with a total radiation dosage of $\sim 68.7 \mu \mathrm{Sv}(\approx 2 \%$ of a standard neck CT, which is $3 \mathrm{mSv}$ ).

Before data collection, each participant was asked to practice all of the movements several times under the guidance of an experienced instructor until s/he was able to perform the motions without any guidance. During head F-E, participants bent their heads first forward and then backward to maximum stretch positions without discomfort. During head LB, they bent their heads downward toward the left shoulder and then downward toward the right shoulder to maximal positions without discomfort. During head AR, they rotated their heads 

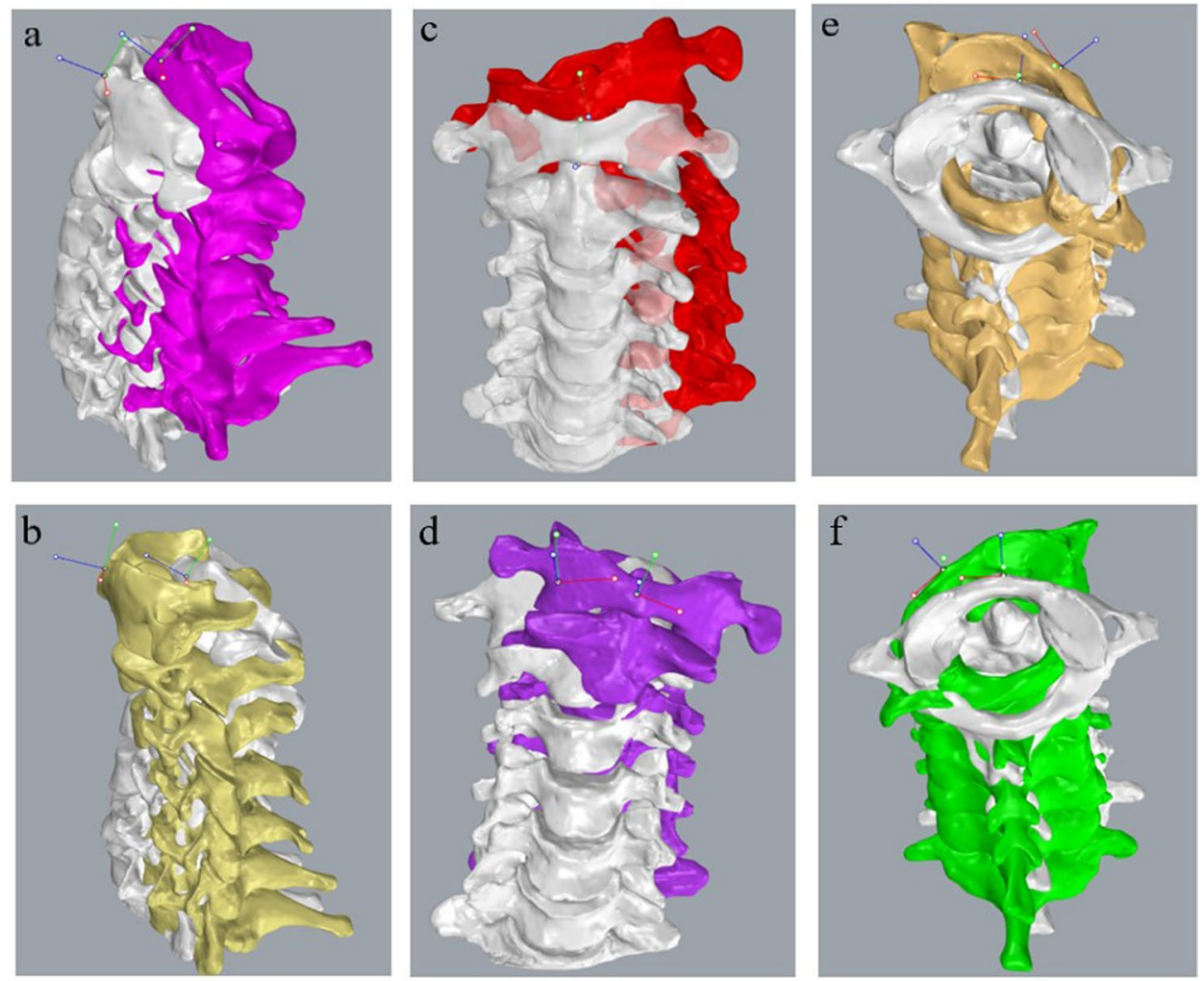

Figure 2. Three-dimensional (3D) models of the cervical spine of a representative healthy young male subject. (a,b) F-E models. (c,d) LB models. (e,f) AR models. The functional range-of-motion positions are pseudocolored and shown together with a grey $3 \mathrm{D}$ model representing the neutral position.

first leftward and then rightward to maximal rotation positions without discomfort. After each stretch, the participants returned their heads to a neutral position. The patients took CT scans in conventional supine position. They took CBCT scans in sitting position.

Model registration. In the first phase, automated registration technology (ART) was used to superimpose each participant's CT segmental model onto its corresponding CBCT segmental model in the neutral position (Fig. 1) using Fluo-Motion software (v1.0, Innomotion Inc., Shanghai, China). In the second phase, ART was used to superimpose the neutral segmental model onto each corresponding end position of the movement segmental models in Fluo-Motion software. A 3D-3D cervical segmental model registration was performed based on iterative closest point method ${ }^{21}$. Each moving cervical segmental model was translated and rotated iteratively in space to align its corresponding target model in the neutral position. The summation of perpendicular distances of moving model vertices was calculated with respect to the closest target model surfaces. Optimization alignment was obtained when the summation reached minimum.

Kinematic analysis. To describe 3D segmental motion characteristics, each model was embedded in an anatomic coordinate system defined by vertebra features ${ }^{22}$ (Fig. 3 ). The coordinate system originated at the most posterior-inferior point of the vertebral body in the mid-sagittal plane, with a left-going positive $\mathrm{x}$ axis, the positive $\mathrm{y}$ axis directed superiorly, and the positive $\mathrm{z}$ axis directed anteriorly. Intervertebral joint angles were determined based on the rotation of the coordinate system of each vertebra relative to that of the subjacent vertebra. Intervertebral translations were similarly defined as the relative displacement of the origins. We used $\mathrm{mm}$ for translation and $\operatorname{deg}\left({ }^{\circ}\right)$ for rotation.

\section{Results}

Mean deviations of CBCT models from their corresponding superimposed CT models (range 0.30-0.57 mm) are reported in Table 1 . The mean deviation ranges of the six end positions of functional movements from the neutral position for the CBCT model were 0.14-0.67 mm during head F-E (Table 2), 0.15-0.66 mm during LB (Table 3), and 0.14-0.65 mm during head AR (Table 4). The local fit levels of the models are indicated with a color-bar in Fig. 4. Usually, maximum deviations occurred at the tip of the transverse process or bony structure edges, which should have little influence on overall kinematic analyses.

Our in vivo cervical spine kinematic data are presented in Figs. 5 and 6. During head F-E, at the C1-C2 level, the primary flexion and primary extension extents were $4.03 \pm 5.81^{\circ}$ and $7.89 \pm 4.78^{\circ}$, respectively. Translation in the three examined directions were generally $<1 \mathrm{~mm}$, coupled with LB and AR that were $<1^{\circ}$ (Table 2). During 


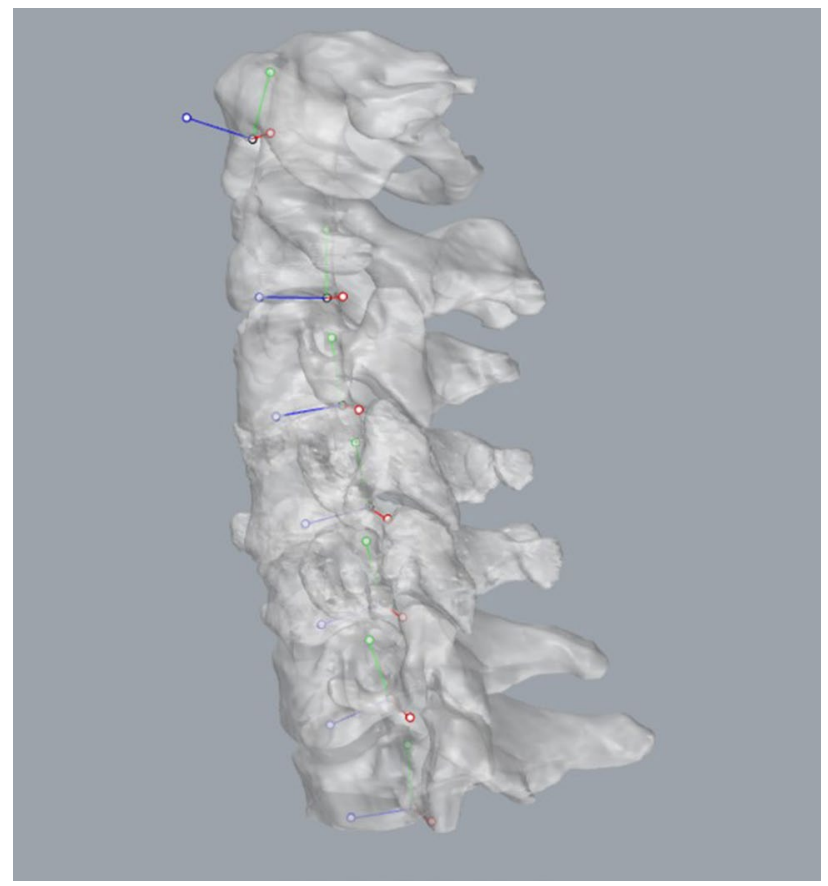

Figure 3. Schematic of the anatomic coordinate system employed in this study. Red arrows show F-E axes, blue arrows show $\mathrm{LB}$ axes, and green arrows show AR axes.

\begin{tabular}{|c|c|c|c|c|c|c|c|c|}
\hline \multirow[b]{2}{*}{ Level } & \multicolumn{4}{|l|}{ Average distance } & \multicolumn{2}{|c|}{$\begin{array}{l}\text { Maximum } \\
\text { distance }\end{array}$} & \multirow[b]{2}{*}{ Standard deviation } & \multirow[b]{2}{*}{ Angular prediction } \\
\hline & Mean deviation & Mean absolute deviation & + & - & + & - & & \\
\hline $\mathrm{C} 1$ & 0.03 & 0.30 & 0.33 & -0.27 & 3.42 & -3.52 & 0.41 & 0.14 \\
\hline $\mathrm{C} 2$ & 0.02 & 0.24 & 0.25 & -0.22 & 2.23 & -2.46 & 0.30 & 0.10 \\
\hline $\mathrm{C} 3$ & 0.06 & 0.24 & 0.29 & -0.18 & 2.31 & -2.05 & 0.32 & 0.30 \\
\hline $\mathrm{C} 4$ & 0.06 & 0.25 & 0.30 & -0.19 & 2.28 & -2.36 & 0.35 & 0.30 \\
\hline C5 & 0.06 & 0.26 & 0.32 & -0.20 & 2.48 & -2.46 & 0.36 & 0.30 \\
\hline C6 & 0.04 & 0.29 & 0.32 & -0.25 & 2.70 & -2.74 & 0.39 & 0.20 \\
\hline C7 & 0.05 & 0.39 & 0.34 & -0.44 & 3.13 & -3.20 & 0.57 & 0.24 \\
\hline
\end{tabular}

Table 1. Accuracy of CBCT models of cervical spine vertebrae relative to CT models. Units, mm, ${ }^{\circ}$.

head LB, the extent of translation in the three ordinate directions were mostly $<1 \mathrm{~mm}$ and most of the movement occurred at the $\mathrm{C} 1-\mathrm{C} 2$ level, with mean displacements of $17.35 \pm 2.38^{\circ}$ leftward and $15.68 \pm 7.03^{\circ}$ rightward (Table 3). Head LB was coupled with AR in the direction opposite to the direction of LB at the C1-C2 level as well as minimal F-E motions at subaxial cervical levels. During the head AR assessment, we found that the mean primary AR extents at the $\mathrm{C} 1-\mathrm{C} 2$ level were $29.93 \pm 7.19^{\circ}$ for leftward rotation and $31.38 \pm 8.49^{\circ}$ for rightward rotation with some concomitant LB in the direction opposite to that of the AR coupled with extension (Table 4). At subaxial cervical levels, AR was coupled with LB in the same direction as the AR movement.

\section{Discussion}

The present study introduces and provides validation of a combined CBCT imaging with 3D-3D registration technique for in vivo measurement of 6-DOF of cervical spine motions. We validated the accuracy of CBCT models relative to CT models, and then confirmed the accuracy of our CBCT data across six positions (F-E, AR left and right, and LB left and right), relative to neutral-position CBCT models. The present data show that the presently validated technique is feasible for analyses of the cervical spine over a wide range of motions.

We used a CT imaging reference model because CT images are known to be highly accurate ${ }^{1,13,14,23}$. Previously, Lim et al. ${ }^{24}$ used CT images of two cervical spines to show that a 3D spinal motion analysis method was accurate within $1 \mathrm{~mm}$ of translation and $1^{\circ}$ of rotation. The accuracy of $3 \mathrm{D}$ surface models is dependent on several technical parameters, including detector sensitivity, x-ray beam inhomogeneity, and reconstruction technique limitations ${ }^{25}$. Spatial accuracy of CBCT specifically tends to be higher at the center of the volume than at the margins ${ }^{26}$. The present data indicate that mean deviations of CBCT imaged structures relative to CT images were in the range of $0.30-0.57 \mathrm{~mm}$. For all six end positions of functional cervical spine movements evaluated in this 


\begin{tabular}{|c|c|c|c|c|c|c|c|c|c|}
\hline \multirow[b]{2}{*}{ Posture } & \multirow[b]{2}{*}{ Level } & \multicolumn{4}{|l|}{ Average distance } & \multicolumn{2}{|c|}{$\begin{array}{l}\text { Maximum } \\
\text { distance }\end{array}$} & \multirow[b]{2}{*}{ Standard deviation } & \multirow[b]{2}{*}{ Angular prediction } \\
\hline & & Mean deviation & $\begin{array}{l}\text { Mean absolute } \\
\text { deviation }\end{array}$ & + & - & + & - & & \\
\hline \multirow{7}{*}{ Flexion } & $\mathrm{C} 1$ & 0.02 & 0.21 & 0.22 & -0.19 & 3.20 & -2.90 & 0.31 & 0.10 \\
\hline & $\mathrm{C} 2$ & 0.01 & 0.18 & 0.17 & -0.19 & 2.23 & -2.63 & 0.24 & 0.05 \\
\hline & C3 & 0.02 & 0.18 & 0.16 & -0.20 & 1.84 & -2.08 & 0.22 & 0.10 \\
\hline & $\mathrm{C} 4$ & 0.02 & 0.21 & 0.19 & -0.23 & 1.96 & -2.12 & 0.24 & 0.10 \\
\hline & $\mathrm{C} 5$ & 0.03 & 0.18 & 0.16 & -0.22 & 1.38 & -2.31 & 0.22 & 0.14 \\
\hline & C6 & 0.00 & 0.19 & 0.19 & -0.19 & 1.92 & -2.56 & 0.25 & 0.00 \\
\hline & $\mathrm{C} 7$ & 0.04 & 0.38 & 0.42 & -0.34 & 3.62 & -3.39 & 0.67 & 0.20 \\
\hline \multirow{7}{*}{ Extension } & $\mathrm{C} 1$ & 0.01 & 0.19 & 0.18 & -0.20 & 2.85 & -3.35 & 0.31 & 0.05 \\
\hline & $\mathrm{C} 2$ & 0.00 & 0.13 & 0.13 & -0.13 & 1.90 & -2.19 & 0.19 & 0.00 \\
\hline & $\mathrm{C} 3$ & 0.02 & 0.09 & 0.11 & -0.07 & 1.76 & -2.10 & 0.16 & 0.10 \\
\hline & $\mathrm{C} 4$ & 0.02 & 0.09 & 0.11 & -0.07 & 1.34 & -1.32 & 0.14 & 0.10 \\
\hline & C5 & 0.02 & 0.11 & 0.12 & -0.09 & 1.97 & -1.56 & 0.15 & 0.10 \\
\hline & C6 & 0.01 & 0.12 & 0.12 & -0.11 & 2.48 & -2.50 & \begin{tabular}{|l|}
0.18 \\
\end{tabular} & \begin{tabular}{|l|}
0.05 \\
\end{tabular} \\
\hline & C7 & \begin{tabular}{|l|}
0.02 \\
\end{tabular} & 0.21 & \begin{tabular}{|l|}
0.22 \\
\end{tabular} & -0.19 & 3.20 & -2.90 & \begin{tabular}{|l|}
0.31 \\
\end{tabular} & 0.10 \\
\hline
\end{tabular}

Table 2. Accuracy of CBCT-modeled cervical spine vertebrae across functional E-F position models relative to a neutral position model. Units, $\mathrm{mm},{ }^{\circ}$.

\begin{tabular}{|c|c|c|c|c|c|c|c|c|c|}
\hline \multirow[b]{2}{*}{ Posture } & \multirow[b]{2}{*}{ Level } & \multicolumn{4}{|l|}{ Average distance } & \multicolumn{2}{|c|}{$\begin{array}{l}\text { Maximum } \\
\text { distance }\end{array}$} & \multirow[b]{2}{*}{$\begin{array}{l}\text { Standard } \\
\text { deviation }\end{array}$} & \multirow[b]{2}{*}{$\begin{array}{l}\text { Angular } \\
\text { prediction }\end{array}$} \\
\hline & & Mean deviation & $\begin{array}{l}\text { Mean absolute } \\
\text { deviation }\end{array}$ & + & - & + & - & & \\
\hline \multirow{7}{*}{ Right bend } & $\mathrm{C} 1$ & 0.00 & 0.19 & 0.19 & -0.19 & 3.22 & -2.37 & 0.33 & 0.00 \\
\hline & $\mathrm{C} 2$ & 0.00 & 0.14 & 0.14 & -0.14 & 2.07 & -2.56 & 0.23 & 0.00 \\
\hline & $\mathrm{C} 3$ & 0.00 & 0.14 & 0.14 & -0.14 & 1.96 & -2.23 & 0.20 & 0.00 \\
\hline & $\mathrm{C} 4$ & 0.02 & 0.14 & 0.12 & -0.15 & 1.90 & -2.47 & 0.17 & 0.10 \\
\hline & C5 & 0.02 & 0.12 & 0.10 & -0.13 & 1.80 & -2.02 & 0.15 & 0.10 \\
\hline & C6 & 0.00 & 0.11 & 0.11 & -0.11 & 1.46 & -2.34 & 0.16 & 0.00 \\
\hline & $\mathrm{C} 7$ & 0.03 & 0.40 & 0.43 & -0.37 & 3.29 & -3.43 & 0.64 & 0.14 \\
\hline \multirow{7}{*}{ Left bend } & $\mathrm{C} 1$ & 0.01 & 0.19 & 0.18 & -0.19 & 2.30 & -2.77 & 0.28 & 0.05 \\
\hline & $\mathrm{C} 2$ & 0.01 & 0.16 & 0.15 & -0.16 & 2.11 & -2.43 & 0.22 & 0.05 \\
\hline & C3 & 0.02 & 0.17 & 0.18 & -0.15 & 2.04 & -2.21 & 0.24 & 0.10 \\
\hline & $\mathrm{C} 4$ & 0.01 & 0.13 & 0.12 & -0.14 & 1.87 & -2.29 & 0.18 & 0.05 \\
\hline & C5 & 0.01 & 0.14 & 0.13 & -0.14 & 2.14 & -2.30 & 0.18 & 0.05 \\
\hline & C6 & 0.01 & 0.13 & 0.13 & -0.12 & 1.44 & -1.72 & \begin{tabular}{|l|}
0.18 \\
\end{tabular} & \begin{tabular}{|l|l|}
0.05 \\
\end{tabular} \\
\hline & C7 & 0.01 & \begin{tabular}{|l|}
0.44 \\
\end{tabular} & 0.45 & -0.43 & 3.20 & -3.34 & \begin{tabular}{|l|l|}
0.66 \\
\end{tabular} & \begin{tabular}{|l|l|}
0.05 \\
\end{tabular} \\
\hline
\end{tabular}

Table 3. Accuracy of CBCT-modeled cervical spine vertebrae across functional LB position models relative to a neutral position model. Units, $\mathrm{mm},{ }^{\circ}$.

study, the mean deviations relative to the neutral model were in the range of $0.14-0.67 \mathrm{~mm}$ and located mostly near the periphery of the vertebrae. The sites of maximum deviation were the tips of transverse processes, spinous processes, and the bony edges that form facet joints, which have little influence on overall accuracy in kinematic analyses. To augment our model registration accuracy, we used an automated surface-matching algorithm that employs point-based registration ${ }^{27}$. The main advantage of this method is that the entire $3 \mathrm{D}$ model surface is used for registration, rather than a predefined set of landmarks ${ }^{28}$. Although we obtained similarly accurate results with CT- and CBCT-based measurements, the CBCT method has several noteworthy benefits, including a low radiation dose ( $\sim 2 \%$ of a regular neck CT exam) and an ability to provide clinically relevant information as well as novel 3D data for research ${ }^{29}$.

Previously, $\mathrm{CT}^{1,14,15}, \mathrm{MRI}^{12}$, and dual fluoroscopy ${ }^{30-32}$ techniques have been employed to obtain in vivo cervical spine measurements. Notably, Nagamoto et al. ${ }^{12}$ used MRI to compare cervical spine kinematics during head rotation between patients with cervical spondylopathy and an asymptomatic control group. However, MRI scans are time-intensive and MRI studies do not reflect the biomechanics of the spine under physiological loading because subjects are scanned in a supine position. Wang et $\mathrm{al}^{30}$ investigated the ranges of motion of the three joints in the cervical spines (from C3 to C7) using biplane fluoroscopic imaging. However, due to skull occlusion, the general biplane technique has not reported the movement of the upper cervical spine. To the best 


\begin{tabular}{|c|c|c|c|c|c|c|c|c|c|}
\hline \multirow[b]{2}{*}{ Posture } & \multirow[b]{2}{*}{ Level } & \multicolumn{4}{|l|}{ Average distance } & \multicolumn{2}{|c|}{$\begin{array}{l}\text { Maximum } \\
\text { distance }\end{array}$} & \multirow[b]{2}{*}{$\begin{array}{l}\text { Standard } \\
\text { deviation }\end{array}$} & \multirow[b]{2}{*}{$\begin{array}{l}\text { Angular } \\
\text { prediction }\end{array}$} \\
\hline & & Mean deviation & $\begin{array}{l}\text { Mean absolute } \\
\text { deviation }\end{array}$ & + & - & + & - & & \\
\hline \multirow{7}{*}{ Right rotation } & $\mathrm{C} 1$ & 0.00 & 0.19 & 0.20 & -0.20 & 2.41 & -2.49 & 0.29 & 0.00 \\
\hline & $\mathrm{C} 2$ & 0.00 & 0.14 & 0.15 & -0.14 & 2.32 & -1.82 & 0.21 & 0.00 \\
\hline & $\mathrm{C} 3$ & 0.00 & 0.14 & 0.13 & -0.14 & 1.75 & -1.86 & 0.18 & 0.00 \\
\hline & $\mathrm{C} 4$ & 0.02 & 0.14 & 0.10 & -0.11 & 1.71 & -1.28 & 0.14 & 0.10 \\
\hline & $\mathrm{C} 5$ & 0.02 & 0.12 & 0.11 & -0.11 & 1.76 & -1.76 & 0.15 & 0.10 \\
\hline & C6 & 0.00 & 0.11 & 0.12 & -0.12 & 1.58 & -2.32 & 0.18 & 0.00 \\
\hline & $\mathrm{C} 7$ & 0.03 & 0.40 & 0.29 & -0.35 & 3.07 & -3.59 & 0.52 & 0.14 \\
\hline \multirow{7}{*}{ Left rotation } & $\mathrm{C} 1$ & 0.01 & 0.19 & 0.18 & -0.19 & 2.25 & -2.46 & 0.27 & 0.05 \\
\hline & $\mathrm{C} 2$ & 0.00 & 0.15 & 0.15 & -0.15 & 2.19 & -1.88 & 0.21 & 0.00 \\
\hline & $\mathrm{C} 3$ & 0.00 & 0.13 & 0.13 & -0.13 & 1.58 & -1.95 & 0.17 & 0.00 \\
\hline & $\mathrm{C} 4$ & 0.01 & 0.12 & 0.13 & -0.11 & 1.63 & -1.48 & 0.16 & 0.05 \\
\hline & $\mathrm{C} 5$ & 0.02 & 0.12 & 0.13 & -0.10 & 1.99 & -1.80 & 0.16 & 0.10 \\
\hline & C6 & 0.00 & 0.12 & 0.12 & -0.12 & 2.25 & -2.58 & \begin{tabular}{|l|}
0.18 \\
\end{tabular} & 0.00 \\
\hline & C7 & 0.07 & 0.43 & \begin{tabular}{|l|}
0.36 \\
\end{tabular} & -0.50 & 3.22 & -3.61 & 0.65 & 0.34 \\
\hline
\end{tabular}

Table 4. Accuracy of CBCT-modeled cervical spine vertebrae across functional AR position models relative to a neutral position model. Units, $\mathrm{mm},{ }^{\circ}$.
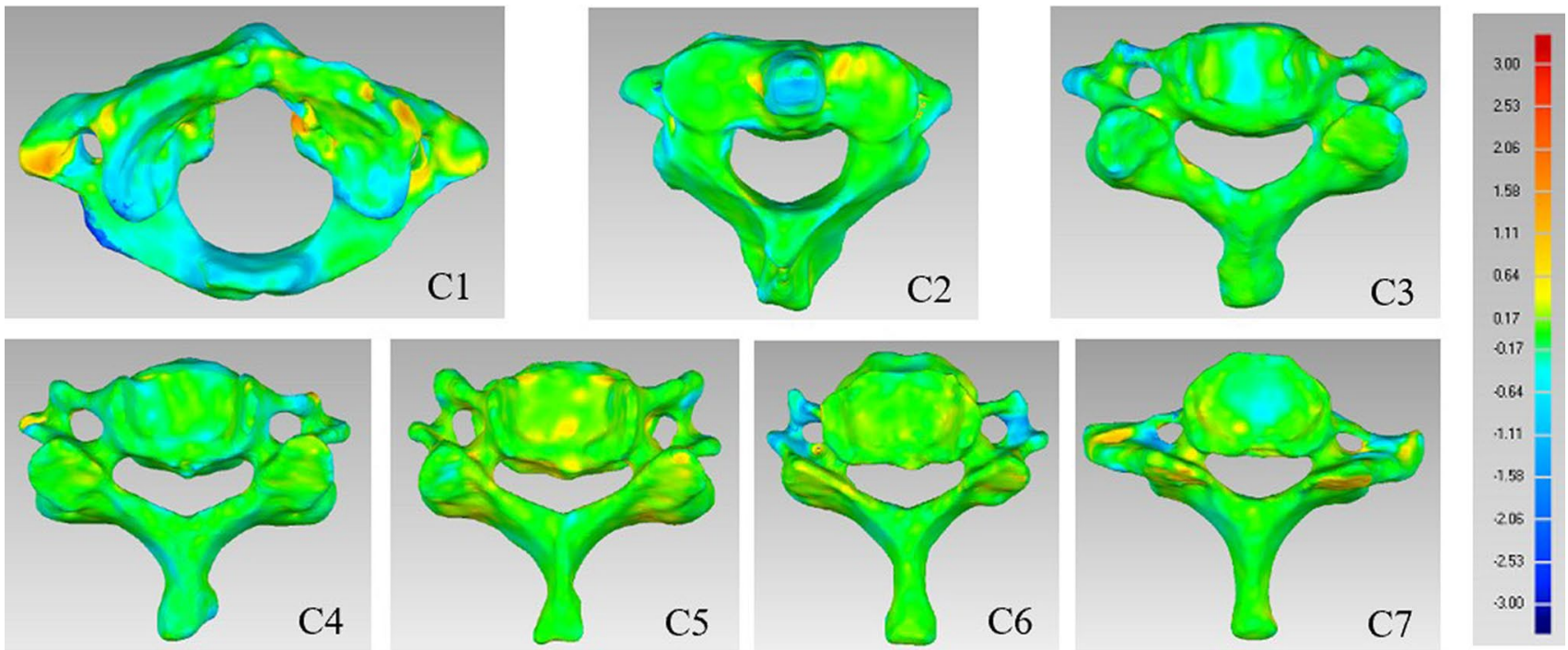

Figure 4. Superimposition of CBCT models with corresponding CT models of each cervical vertebra in the neutral position. Deviations between the CBCT and CT models are indicated with the color bar. Units, $\mathrm{mm}$.

of our knowledge, there are no previously published in vivo CBCT studies that investigated upper and subaxial cervical spine kinematics during F-E, LB, and AR of the head.

In agreement with prior studies ${ }^{14,33}$, the 6-DOF kinematic data reported herein indicate that intervertebral translational motions in ordinal directions are quite small during movements (mostly $<1 \mathrm{~mm})$. Our data also show that most of the rotation of the head in AR occurs between $\mathrm{C} 1$ and $\mathrm{C} 2\left(31.66^{\circ}\right)$, consistent with previous data $^{33,34}$. This property can be attributed to the specialized anatomy of the upper cervical spine, including double convex joints in the lateral parts of $\mathrm{C} 1$ and $\mathrm{C} 2$ as well as the unique odontoid process (a.k.a. dens) on the $\mathrm{C} 2$ vertebra around which the $\mathrm{C} 1$ vertebra rotates, providing coupling mechanics that differ from those that occur at underlying levels. Upper cervical spine movements have complicated 3D mechanics consisting of both a main motion and coupled motions. In this study, we documented coupled LB at $\mathrm{C} 1-\mathrm{C} 2$ that was in the direction opposite to head F-E and AR movements. At subaxial cervical levels, we observed AR-coupled LB in the same direction as that of the AR. These patterns of motion are consistent with those first described by Panjabi et al. ${ }^{35}$ and confirmed in Ishii et al.s ${ }^{33} 3 \mathrm{D}$ kinematic study.

Angular deviation analysis will greatly help to understand the findings in phase 2. As CBCT and CT scans were not performed simultaneously, it is not suitable to directly compare the angular deviation as the postures in two scans may be slightly different. Considering the geometric relationship between linear deviation and angular deviation, a proper estimation would be using the cervical spine dimension. The mean deviation $(0.02-0.06 \mathrm{~mm})$, 

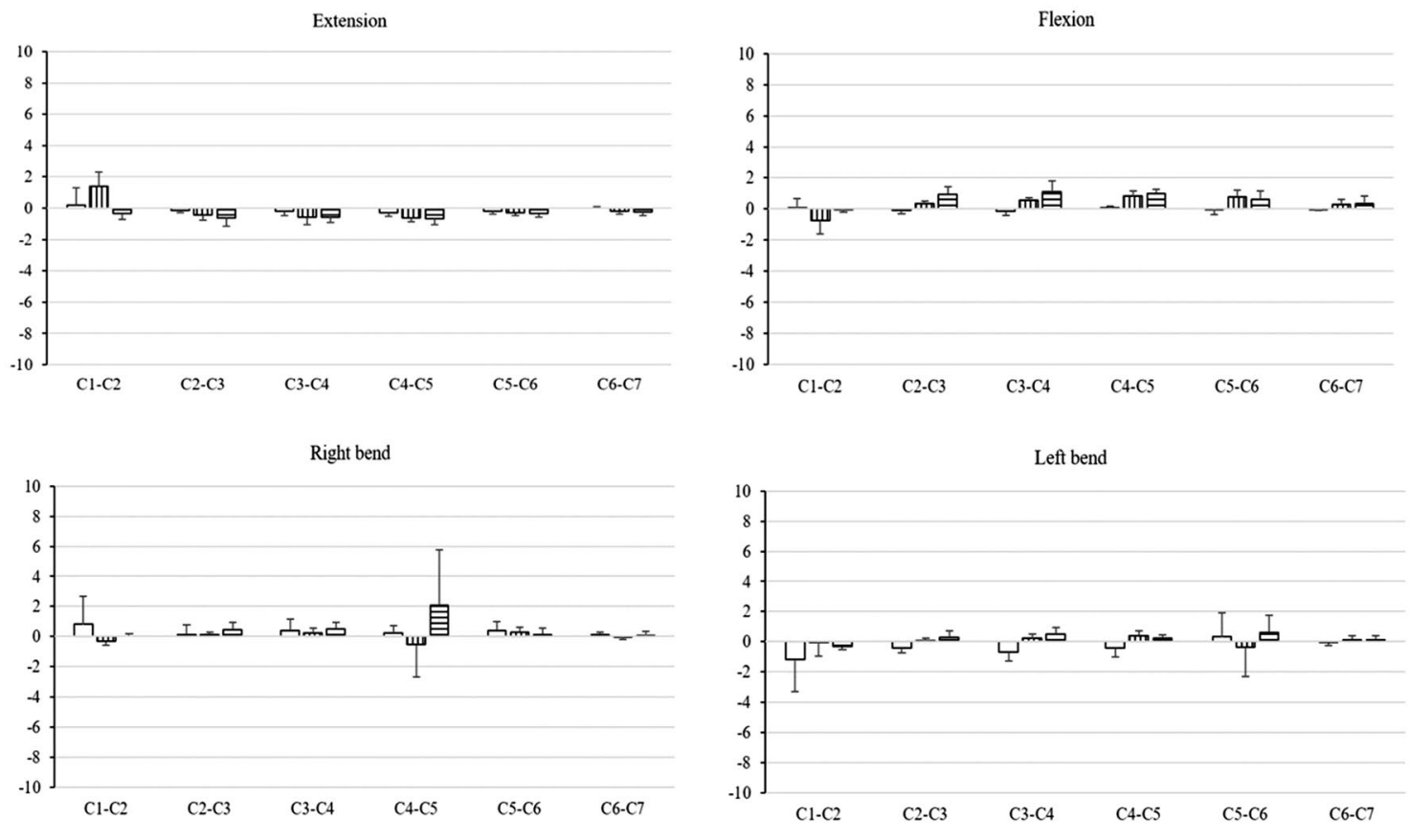

Right rotation
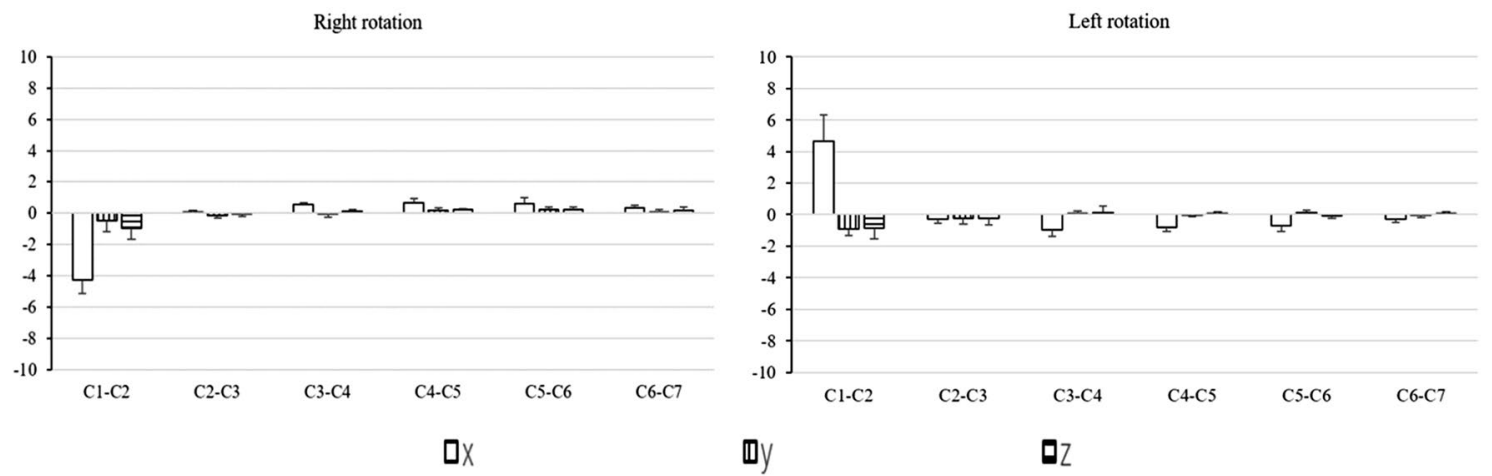

Figure 5. Translation ( $\mathrm{mm})$ changes of the cervical spine at six analyzed positions. $x$, right (-)-left $(+)$; $y$, inferior (-)-superior $(+)$; z, posterior (-)-Anterior (+). A graphic legend indicating the meaning of the bar colors is shown in the bottom right of the figure.

taking into account a worst case over $11.9 \mathrm{~mm}$ cervical dimension (Table 5), will corresponding to $1 / 500$ to $1 / 200$ radius, which is approximately $0.1^{\circ}$ to $0.3^{\circ}$. The proposed CBCT kinematic analysis can be used as an assisted diagnosis tool for patients without instrumentation. However, as a next step, it would also be a potential tool for the evaluation of surgery. We checked several post-op patients with instrumentation and found little metal artifact in CBCT scans (Appendix Fig. 1). In addition, similar mean absolute deviation of 0.2-0.3 mm were also found with respect to those without instrumentation (Appendix Fig. 2). In the future, with larger sample size, we will report kinematic analysis of post-op patients to investigate fixation and influence to adjacent levels.

Several limitations of this study should be noted. Firstly, although CBCT radiation doses are lower than those of traditional CT, there is still some amount of radiation exposure. In future studies, protocols can be modified according to particular circumstances to further reduce radiation dose when possible, such as when spine motion does not need to be measured in all directions or by measuring upper or subaxial motions separately. Secondly, because our primary aim was to validate this technique with 10 subjects, only 5 additional healthy subjects were tested to verify clinical feasibility. We will pursue follow-up studies with additional healthy and pathological subjects. Third, the proposed method is suitable for patients with degenerative disease. Those with severe injury that cannot hold still may not took CBCT scans in seated positions. Nonetheless, the results of this study provide insights into the in vivo 3D mechanics of upper and subaxial cervical spine kinematics captured in human subjects enacting positions.

\section{Conclusion}

The presently examined low-radiation CBCT modeling technique with 3D-3D registration was demonstrated to be valid for obtaining accurate measurements of cervical spine kinematics in vivo. The method yielded measurements of cervical spine vertebrae in six positions at the maximal extents of F-E, LB, and AR of the head with submillimeter deviations from reference models. Because this technique reproduces functional spine positions 

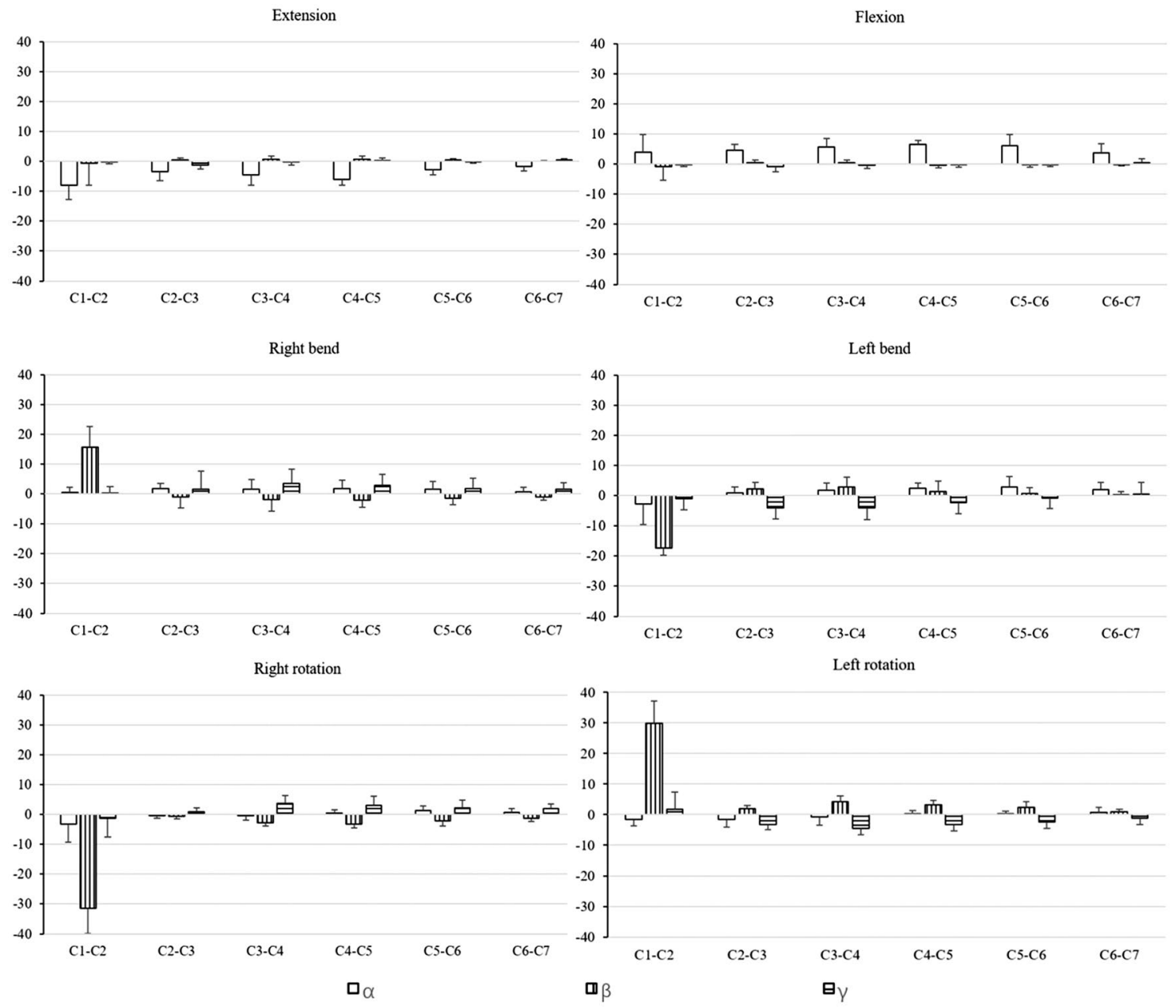

Figure 6. Rotation $\left({ }^{\circ}\right)$ changes of the cervical spine at six analyzed positions. Rotation $\left(^{\circ}\right): \alpha, F(-)$-E $(+) ; \gamma, \mathrm{LB}$ left (-)-right (+); $\beta$, AR right (-)-left (+). A graphic legend indicating the meaning of the bar colors is shown in the bottom right of the figure.

\begin{tabular}{|l|l|l|l|}
\hline & Height $(\mathbf{m m})$ & Width $(\mathbf{m m})$ & Length $(\mathbf{m m})$ \\
\hline C1 & 12.8 & 40.0 & 75.9 \\
\hline C2 & 37.4 & 43.8 & 56.8 \\
\hline C3 & 14.1 & 40.7 & 52.2 \\
\hline C4 & 13.5 & 36.9 & 47.0 \\
\hline C5 & 11.9 & 37.4 & 52.7 \\
\hline C6 & 11.9 & 49.8 & 56.5 \\
\hline C7 & 15.0 & 55.9 & 78.7 \\
\hline
\end{tabular}

Table 5. Typical 3D dimensions of cervical vertebrae.

with 3D anatomic models, 6-DOF cervical spine kinematics can be obtained. The technique can be used for segmental kinematic analysis of the cervical spines, especially at the end positions of functional movements.

Received: 26 June 2021; Accepted: 12 October 2021

Published online: 20 December 2021

\section{References}

1. Kang, J. et al. In vivo three-dimensional kinematics of the cervical spine during maximal active head rotation. PLoS ONE 14, 4 (2019). 
2. Webb, R. et al. Prevalence and predictors of intense, chronic, and disabling neck and back pain in the UK general population. Spine 28(11), 1195 (2003).

3. Zhao, X. et al. Three-dimensional analysis of cervical spine segmental motion in rotation. Arch. Med. Sci. 9, 515-520 (2013).

4. Liu, F. et al. In vivo evaluation of dynamic characteristics of the normal, fused, and disc replacement cervical spines. Spine 32(23), 2578-2584 (2007).

5. McDonald, C. P. et al. Three-dimensional motion analysis of the cervical spine for comparison of anterior cervical decompression and fusion versus artificial disc replacement in 17 patients: clinical article. J. Neurosurg. Spine 20(3), 245-255 (2014).

6. Wang S, Kozanek M, Wood KB et al (2010) Lumbar Degenerative Disc Disease Increases Deformations at Cephalad Adjacent Levels In Vivo. Asme Summer Bioengineering Conference

7. Rabin, D. et al. The kinematics of anterior cervical discectomy and fusion versus artificial cervical disc: A pilot study. Neurosurgery 3, 104-105 (2007).

8. Wang, S. et al. How does lumbar degenerative disc disease affect the disc deformation at the cephalic levels in vivo?. Spine 36(9), E574-E581 (2011)

9. Wang, S. et al. Measurement of geometric deformation of lumbar intervertebral discs under in-vivo weightbearing condition. J. Biomech. 42(6), 705-711 (2009).

10. Schwab, J. S., Diangelo, D. J. \& Foley, K. T. Motion compensation associated with single-level cervical fusion: Where does the lost motion go?. Spine 31(21), 2439 (2006).

11. Ragab, A. A., Escarcega, A. J. \& Zdeblick, T. A. A quantitative analysis of strain at adjacent segments after segmental immobilization of the cervical spine. J. Spinal Disord. Tech. 19(6), 407-410 (2006).

12. He, X. et al. Unsupervised boundary delineation of spinal neural foramina using a multi-feature and adaptive spectral segmentation. Med. Image Anal. 36, 22-40 (2017).

13. Sato, T. \& Masui, K. Morphologic differences in intervertebral foramina: A radiographic study of cervical spine positions in asymptomatic men. J. Manipulative Physiol. Ther. 36(5), 327-332 (2013).

14. Nagamoto, Y. et al. In vivo three-dimensional kinematics of the cervical spine during head rotation in patients with cervical spondylosis. Spine 36(10), 778-783 (2011).

15. Sugiura, T. et al. In vivo 3D kinematics of the upper cervical spine during head rotation in rheumatoid arthritis. J. Neurosurg. Spine 20(4), 404-410 (2014).

16. Salem, W. et al. Invivo three-dimensional kinematics of the cervical spine during maximal axial rotation. Man. Ther. 18(4), 339-344 (2013).

17. Zhu, Z. et al. The accuracy and repeatability of an automatic 2D-3D fluoroscopic image-model registration technique for determining shoulder joint kinematics. Med. Eng. Phys. 34, 9 (2012).

18. Bonfim, M. A. et al. Cervical vertebrae maturation index estimates on cone beam CT: 3D reconstructions vs sagittal sections. Dentomaxillofac. Radiol. 45(1), 2015-2162 (2016).

19. Zhao, C. et al. Cone-beam imaging with tilted rotation axis: Method and performance evaluation. Med. Phys. 47(8), 3305-3320 (2020).

20. Shi, H. \& Scarfe, W. C. Three-dimensional reconstruction of individual cervical vertebrae from cone-beam computed-tomography images. Am. J. Orthod. Dentofac. Orthop. 131(3), 426-432 (2007).

21. Ge, Y., Maurer, C. R. \& Fitzpatrick, J. M. Surface-based 3D image registration using the iterative closest-point algorithm with a closest-point transform. Proc. SPIE Int. Soc. Opt. Eng. 2710, 358-367 (1996).

22. Ishii, T. et al. Kinematics of the cervical spine in lateral bending: In vivo three-dimensional analysis. Spine 31(2), 155-160 (2006).

23. Simon, S. et al. CT imaging techniques for describing motions of the cervicothoracic junction and cervical spine during flexion, extension, and cervical traction. Spine 31(1), 44-50 (2006).

24. Lim, T. H. et al. A noninvasive, three-dimensional spinal motion analysis method. Spine 22, 1996-2000 (1997).

25. Loubele, M. et al. A comparison of jaw dimensional and quality assessments of bone characteristics with cone-beam CT, spiral tomography, and multi-slice spiral CT. Int. J. Oral Maxillofac. Implants 22(3), 446 (2007).

26. Eggers, G. et al. Geometric accuracy of digital volume tomography and conventional computed tomography. Br. J. Oral Maxillofac. Surg. 46(8), 639-644 (2008).

27. Wang, S. et al. Measurement of vertebral kinematics using noninvasive image matching method-validation and application. Spine 33(11), E355 (2008).

28. Liang, X. et al. A comparative evaluation of cone beam computed tomography (CBCT) and multi-slice CT (MSCT). Part II: On 3D model accuracy. Eur. J. Radiol. 75(2), 265-269 (2010).

29. Kapila, S. D. \& Nervina, J. M. CBCT in orthodontics: Assessment of treatment outcomes and indications for its use. Dentomaxillofac. Radiol. 44(1), 20140282 (2015).

30. Wang, H. et al. Quantifying the ranges of relative motions of the intervertebral discs and facet joints in the normal cervical spine. J. Biomech. 112, $110023(2020)$.

31. Anderst, W. J. et al. Validation of a noninvasive technique to precisely measure in vivo three-dimensional cervical spine movement. Spine 36(6), E393 (2011).

32. Chang, V. et al. Dynamic measurements of cervical neural foramina during neck movements in asymptomatic young volunteers. Surg. Radiol. Anat. Sra 39(10), 1-10 (2017).

33. Ishii, T. et al. Kinematics of the upper cervical spine in rotation: in vivo three-dimensional analysis. Spine 29, 2 (2004).

34. Pfirrmann, C. W. et al. Functional MR imaging of the craniocervical junction. Correlation with alar ligaments and occipitoatlantoaxial joint morphology: a study in 50 asymptomatic subjects. Schwz Med. Wochenschr. 130(18), 645-651 (2000).

35. Panjabi, M. M. et al. Mechanical properties of the human cervical spine as shown by three-dimensional loaddisplacement curves. Spine 26(24), 2692e700 (2001).

\section{Acknowledgements}

This study was supported by the (China) State's Key project of Research and Development Plan (grant numbers 2018YFF0300504), the Key basic research projects in the Military Commission's Science and Technology Foundation Strengthening Program [grant numbers 2020-JCJQ-ZS-264], the Natural Science Foundation of China (grant numbers 81760410, 81960408), and Key R \& D projects of Jiangxi Province Science and Technology Department [grant numbers 20202BBG73033]. The authors gratefully acknowledge financial support from China Scholarship Council.

\section{Author contributions}

Z.W. conceived of the design; W.W. participated in drafting, writing, and revising the manuscript; S.W. helped to draft, write, and revise the manuscript; W.W., C.L., J.L., J.L., F.T., T.Z., D.W., and L.G. to acquisition and analysis of the data; S.W. and Z.W. provided funding for the study. All authors have read and approved the final version of the manuscript, and agree with the order and presentation of the authors. 


\section{Competing interests}

The authors declare no competing interests.

\section{Additional information}

Supplementary Information The online version contains supplementary material available at https://doi.org/ 10.1038/s41598-021-01319-x.

Correspondence and requests for materials should be addressed to S.W.

Reprints and permissions information is available at www.nature.com/reprints.

Publisher's note Springer Nature remains neutral with regard to jurisdictional claims in published maps and institutional affiliations.

(c) (1) Open Access This article is licensed under a Creative Commons Attribution 4.0 International License, which permits use, sharing, adaptation, distribution and reproduction in any medium or format, as long as you give appropriate credit to the original author(s) and the source, provide a link to the Creative Commons licence, and indicate if changes were made. The images or other third party material in this article are included in the article's Creative Commons licence, unless indicated otherwise in a credit line to the material. If material is not included in the article's Creative Commons licence and your intended use is not permitted by statutory regulation or exceeds the permitted use, you will need to obtain permission directly from the copyright holder. To view a copy of this licence, visit http://creativecommons.org/licenses/by/4.0/.

(C) The Author(s) 2021 